\title{
Abbreviations and Conventions of Usage
}

\author{
Abbreviations \\ CFPC: Ch'üan fa-pao chi \\ CTL: Ching-te ch'üan-teng lu \\ CTW: Ch'ing-ting ch'üan T'ang-wen \\ EJSHL: Erh-ju ssu-hsing lun \\ H: Hsü tsang-ching \\ HKSC: Hsü kao-seng chuan \\ JTFM: Ju-tao an-hsin yao fang-pien fa-men \\ KSC: Kao-seng chuan \\ LCFJC: Leng-ch'ieh jen-fa chih \\ LCSTC: Leng-ch'ieh shih-tzu chi \\ LTFPC: Li-tai fa-pao chi \\ SKSC: Sung kao-seng chuan \\ T: Taishō shinshū dai-zōkyō \\ TCL: $\quad$ Tsung-ching lu \\ TTC: Tsu-t'ang chi \\ Z: $\quad$ Dai Nippon zoku-zōkyō
}

CONVENTIONS OF USAGE

1. Ages of individuals are given in the Chinese style.

2. Terms such as "Buddha Nature" have been rendered capitalized and without hyphenation in order to accomodate occasional abbreviated references to "Nature" alone.

3. Interlineal glosses in translated passages are indicated by the conjoint use of parentheses and italics, i.e., (Another text says . . .).

4. Chapter and section headings of the text are indicated by capitalized Roman numerals and Arabic numerals, respectively, while Roman nu- 
merals and capitalized English letters have been used for the chapter and section headings of translated works.

5. Citations of material in Tun-huang manuscripts are given in the following form: manuscript line number, slash, plate number, colon, plate line number, e.g., 607/26:2.

6. The annotation for the composite version of the $W u$ fang-pien included is explained in $\mathbf{n} .224$ to Section Two.

7. All citations of material from the Zoku-zokyō have been crossreferenced to the Taiwan reprint, the $H$ sü tsang-ching. 